\title{
Proxemic Interaction in a Multi-Room Music System
}

\author{
Henrik Sørensen, Mathies G. Kristensen, Jesper Kjeldskov and Mikael B. Skov \\ Department of Computer Science / Research Centre for Socio-Interactive Design \\ Aalborg University \\ Denmark \\ \{hesor, mathies, jesper, dubois\}@cs.aau.dk
}

\begin{abstract}
In recent years we have seen a growing interest in proxemic interaction within HCI. In order to explore proxemic interaction that spans across separate locations, we have developed a functional prototype of a multiroom music system, called AirPlayer, and performed a field evaluation. The system implements proxemic interactions on top of an existing Apple AirPlay based platform. The added features allow music to follow the user around the house and provide a smartphone app which can adapt to the current location of the user. The prototype was deployed in two households over a three week period, where data was collected through logging, user-written diaries and interviews. What the field evaluation has revealed is a number of interesting findings specifically regarding the importance of a simple interaction, the power of discrete zones to provide a local interaction, the importance and challenge of understanding background interactions and challenges in designing interaction with music in discrete zones.
\end{abstract}

\section{Author Keywords}

Proxemic interaction; music consumption; ubiquitous music; prototype; field evaluation.

\section{ACM Classification Keywords}

H5.m. Information interfaces and presentation (e.g., HCI): Miscellaneous.

\section{INTRODUCTION}

Our homes are getting populated by an increasing number of network enabled devices, technically capable of interacting with each other. This creates new challenges within the field of HCI and work is needed to explore how to take advantage of the possibilities that emerge in the home as a ubiquitous computing (ubicomp) environment.

One way of approaching the interaction design of systems, within ubicomp environments, is through the perspective of proxemics. Hall originally coined the term proxemics as a way of describing interpersonal interaction based on physical measures (Hall, 1966). In his work he defined discrete zones surrounding us, which are meaningful to the way we interact with each other.

Permission to make digital or hard copies of all or part of this work for personal or classroom use is granted without fee provided that copies are not made or distributed for profit or commercial advantage and that copies bear this notice and the full citation on the first page. To copy otherwise, or republish, to post on servers or to redistribute to lists, requires prior specific permission and/or a fee.

$\mathrm{OzCHI} 13$, November 25 - 29 2013, Adelaide, Australia

Copyright 2013 ACM 978-1-4503-2525-7/13/11 ..\$15.00.
The notion of proxemic interaction used in this paper is based on an elaboration of Hall's work, by Greenberg et al. (2011), where they apply proxemics to interaction within ubicomp environments. The purpose is to take advantage of the way proxemics influence how we naturally interact with each other, and apply this knowledge to interaction within ubicomp environments.

Recent work has explored the possibilities of proxemic interactions for both work related contexts ( $\mathrm{Ju}$ et al., 2008; Greenberg et al., 2011) and leisure contexts (Greenberg et al., 2011; Ballendat et al., 2010; Wang et al., 2012; Clark et al., 2011). Most studies provide valuable results on what can be called the micro level of proxemic interaction. An example is where content on a large display changes appropriately according to the distance and orientation of the user, as shown in the Proxemic Media Player (Greenberg et al., 2011). On a larger scale, the macro level, interaction spans multiple locations. In many cases this introduces a real conceptual difference and although the macro level sometimes consists of several micro level systems, it is important to investigate this type of interaction designs as a whole and see how they are used in real-life contexts.

Music consumption is an area where we see a natural application of proxemic interactions. Music plays an important role in many people's lives across age, gender and culture. The digitisation of music, and advances in mobile and network technologies, have opened up for new opportunities in interaction design. Holmquist describes this phenomenon as ubiquitous music (Holmquist, 2005) and several new advances have recently found its way into the consumer market. An example is the emergence of online music services which contains millions of songs, available through a subscription, making it accessible from several different devices. The growing integration of wireless networks, in our homes, has additionally changed the way we listen to and control music at home. Several music systems, such as Sonos, Bose link, Bang \& Olufsen BeoLink and Apple AirPlay, allow us to listen to the same music collection, and use mobile devices to control music, in our entire home. On top of the opportunities offered by this new infrastructure is also consequently an extra layer of complexity for the interaction design. How does the user choose where to play music? How does the user direct control towards a specific location? The same music could of course play in the entire house simultaneously, but what if different persons want to listen to different songs in different rooms? What if the music needs to be louder in a large room and quieter in a small room? 
AirPlayer has been developed as an approach to explore these challenges by hiding the complexity of multi-room music systems through an integration of proxemic interactions. Because music is not directional in the same way as visual content, we furthermore find the multiroom music system an interesting case for specifically studying the location and movement dimensions of proxemic interaction. The user here moves between different locations, and other dimensions such as orientation and distance can be less significant locally. In our work we focus on how an implementation of the proxemic interactions is used during everyday situations. Due to the importance of the context in which the interaction occurs, findings are based on field evaluations conducted in households, over a three week period, as an integrated part of the participants' existing multi-room music system.

In the remaining sections of this paper we present related work to both proxemic interaction and ubiquitous music. We then describe the AirPlayer system in terms of interaction design and system implementation. Lastly, we present the conducted field evaluation and discuss the results.

\section{RELATED WORK}

This section will put our work in relation to existing work to clarify the motivation for exploring proxemic interactions in a music consumption context.

\section{Proxemic Interaction}

In the work of Greenberg et al. (2011) proxemics are operationalized in a way suitable to the interaction within ubicomp environments between people, digital devices, and non-digital things. They break proxemics into five measurable dimensions: Distance, orientation, movement, identity and location. The dimensions provide discrete and/or continuous measures, which can influence the interaction taking place. Work originating from this operationalization of proxemics includes a proximity toolkit for fast prototyping of proxemic interactions (Marquardt et al., 2011), and the use of the sociological constructs, F-formations and micro-mobility, in the design of cross-device interaction (Marquardt et. al., 2012). The five dimensions provide a great framework for exploration of proxemics in various ubicomp contexts and in their work they have furthermore identified six immediate challenges in proxemic interactions design (Marquardt and Greenberg, 2012). These are for example the challenges of directing actions or providing feedback to the user, when the interaction is based on proxemics.

The application of proxemics in HCI has primarily moved towards different aspects in relation to a central device of focus. Vogel and Balakrishnan (2004) have previously specified a set of design principles for public ambient displays. In their work they define a framework, for what they refer to as interaction phases. The idea is that the area in front of the device is divided into four discrete phases similar to Hall's proxemic zones (Hall, 1966) surrounding a person. Each phase is determined by the distance to nearby users and they describe the interaction as transitioning from implicit and public to explicit and personal interaction. Recent work has explored similar applications of proxemics, such as public displays (Wang et al., 2012), whiteboards (Ju et al., 2008) or tabletops (Annett et al., 2011; Ackad et al., 2012). These studies provide important insight into proxemics in relatively small spaces and help us understand how proxemics can facilitate a different interaction form in such ubicomp environments. There has however not been the same focus on proxemics on a larger scale.

The idea of exploring proxemic interactions in larger spaces than a single room, or the immediate area surrounding a display, is of course not entirely new and unexplored. An early, well-known, example is the Active Badge (Want et al., 1992). The Active Badge is aimed at a work context, where employees can be tracked via a wearable badge. The badge contains an infrared beacon from which a sensory network picks up the signal and updates the user position every 15 seconds. Information can for instance be used by receptionists to direct calls to the correct location. The system has successfully been deployed on a large scale at several locations. The UbERBadge (Laibowitz et al., 2006) is a different approach to proxemic aware wearable badges. In this case, the badges acts as sensor nodes used to facilitate social interaction at large meetings. Each badge contains sensors which can detect other badges or stationary tags at well-known locations. Possible applications include locating other badge-wearers, exchanging contact information wirelessly, and as an interaction device at appropriate locations. The EasyLiving project (Brummit et al., 2000) is an example of proxemic interaction in the home, with a focus on an architecture that aggregates devices into a coherent user experience. This is accomplished through technologies that track people and devices, and a middleware application to bind it together.

Proxemic interactions on a larger scale, where interaction spans separate rooms of a house, is closely related to the work on indoor positioning, which is a huge research area within ubicomp. Based on measured physical quantity and hardware technology, indoor positioning technologies can be categorized into radio frequency, photonic, sonic waves, mechanical and others (Torres-Solis et al., 2010). An accurate indoor positioning system is out of the scope of this paper and focus is on the interaction, designed on top of the position system.

\section{Music Consumption}

We find the case of music consumption in a ubicomp environment interesting to proxemic interaction, as the music itself provides immediate non-visual feedback. This is particularly suitable for a case where location and movement is separated from orientation and in some way distance. Holmquist's description of ubiquitous music (Holmquist, 2005) embraces music in interaction design for several research directions. The point is that technological advances have changed the way we listen to music. Liikkanen et al. (2012) encourages a renewed interest within HCI in music interaction. This is both due to the cultural, social and commercial significance of music consumption, but also because music interaction as a research topic has become less visible. 
A radical change in music consumption is the way advances in mobile technologies has enabled us to listen to music on-the-move. In research this has been manifested in a relatively large body of work on mobile music interaction. Recent interesting examples include MusicalHeart which recommends music based on heart rate (Nirjon et al., 2012), and the +++ wearable player (Trotto and Titarelli, 2012) that "infects" passers-by with music while jogging. Ubiquitous music is however not restricted to mobile devices. Integration of wireless network infrastructures in our homes open up for similar novel interaction possibilities. The fact that music has become more ubiquitous does not mean that we have stopped listening to music in fixed locations.

Rose (2000) reflected on music consumption in the home, back in 2000, and suggested an architecture and interaction design with a central music library and a touch interface. A similar use of music as a case for interaction designs in ubicomp environments is seen in the work of Chang and Kim (2006), where a context-aware music playing service is presented. They use a Bluetooth signal to facilitate location recognition, where moving nodes carried by users are detected by a set of fixed nodes. Fixed nodes are then capable of playing music according to the preference of nearby users. What we see is a potential for smarter music systems capable of utilising various devices and a wireless network infrastructure. It is however important also to explore these advances from an HCI perspective.

\section{AIRPLAYER}

AirPlayer has been developed to explore proxemic interaction for a multi-room music system. A multi-room music system, installed throughout the home, allows users to centralise storage and management of their music collection. Remote control of the music can furthermore be integrated into existing devices. An example is a universal smartphone application, replacing different remote controls for each music player placed around the home. This kind of music player platform adds new possibilities but also an additional level of complexity to the interaction. AirPlayer is a multi-room music player integrating proxemic interaction principles, as an approach to take advantage of the new possibilities while hiding the complexity to the user. Allowing the system to be aware of the user's location and movement around the house, enables AirPlayer to infer what part of the house the user wants to interact with and where music should be played. The goal is to explore the use of proxemic interaction as a more implicit form of remote controlling a multi-room music system.

\section{Multi-room Proxemic Interactions}

The proxemic interactions of AirPlayer are implemented as separate features of the system which can be activated independently. The two features, location and movement, are each based on the corresponding dimension from the operationalization of proxemics by Greenberg et al. (2011). In this section, each of the features will be presented through a concrete scenario followed by our interpretation of the dimension in AirPlayer.
Location

The location feature of AirPlayer is activated from the top-left corner of the music player interface as shown in Figure 1.

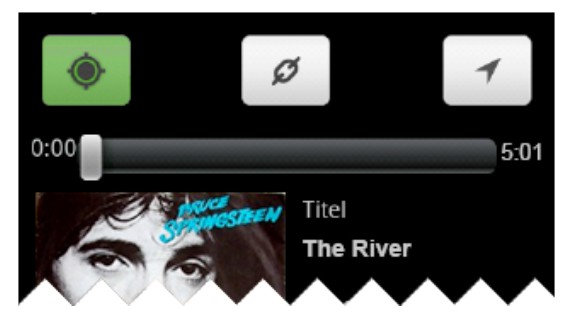

Figure 1. The location feature can be activated on the main screen of the music player with the touch of a button.

It allows the graphical user interface of the smartphone application to adapt to the current location of the user. The following scenario, illustrated in Figure 2 describes a typical use situation.

Alice and Bob enjoy a Sunday evening at home. They are in the living room where Alice is reading a book and Bob is browsing the news on his laptop. Bob has previously used the music browser on the AirPlayer smartphone application to queue a number of songs from their common iTunes library. Because the system knew that he was in the living room, it immediately started playing there as he pressed play. Bob had been up early that morning and goes to the bedroom to get a nap. Bob likes to listen to music as he falls asleep and as a big fan of Bruce Springsteen, he takes out his smartphone to put on his playlist of favourite Bruce Springsteen songs. The smartphone application shows that he is currently in the bedroom and as he selects the playlist, the speakers in the bedroom start playing. The smartphone application shows that it is "The River" playing. Alice is still in the living room listening to the same music as before.
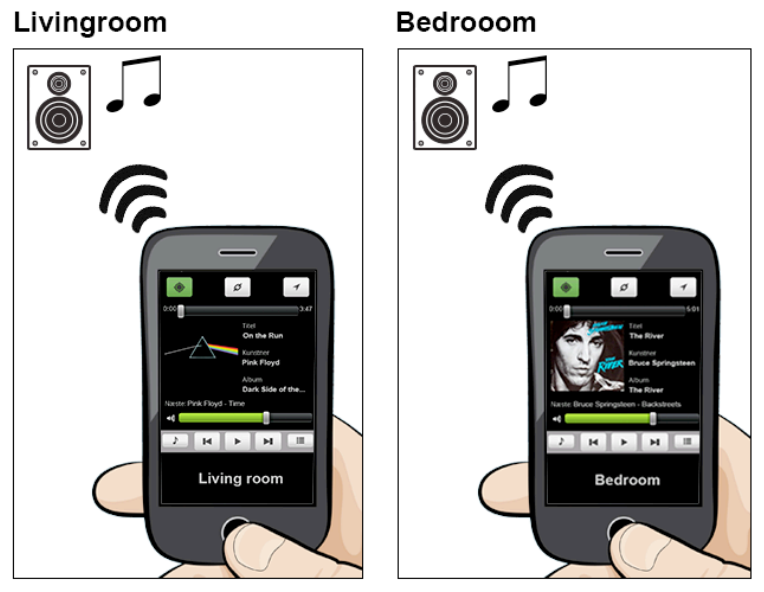

Figure 2. When the location feature is active, the GUI adapts to the current location of the user.

Greenberg et al. (2011) defines the location dimension as a description of the physical context in which the entities reside. Entities in this case can be both people, digital devices and non-digital things. They use a particular room as an example of a distinct location, which is also the 
basis of how it is interpreted in AirPlayer. In AirPlayer the locations are however referred to as zones since the system allows the user to combine separate locations into larger zones. As described in the scenario, the location feature introduces location awareness of the controlling digital device. When Bob is in the living room with his smartphone, AirPlayer knows, and he therefore automatically controls the music in the living room. When he moves to the bedroom, control is automatically applied to the bedroom only, independent of what is playing in the living room. The smartphone application furthermore provides real-time visual feedback to the corresponding zone, showing which zone he is currently in, as well as information about the music playing in that particular zone. Greenberg et al. (2011) puts emphasis on the importance of the location dimension, as the meaning applied to the other dimensions can be dependent on the physical context. In AirPlayer this is the case for the interpretation of the movement dimension.

\section{Movement}

The movement feature is activated from the top-right corner of the music player interface as shown in Figure 3.

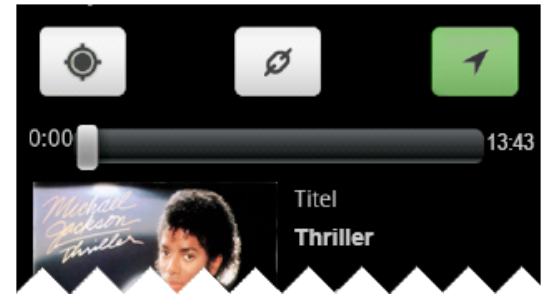

Figure 3. The movement feature is activated in the same way as the location feature on the main screen of the music player.

When activated, it tracks the user's movement around between different locations. This information is used to make music follow the user around continuously. The following scenario, illustrated in Figure 4, explains the use situation.

Charlie is not much of a morning person but music normally helps him get a fresh start of the day. He uses his music system as an alarm clock and at 7:00 AM his stereo starts playing in the bedroom. He grabs his smartphone and goes to the kitchen to get a cup of coffee. The music stops in the bedroom and "follows" him to the kitchen. In the kitchen he suddenly remembers a great song he has not heard in a while. He picks up his smartphone, finds the song in the AirPlayer application, and puts it in the queue. After he finishes his coffee he goes to the bathroom to take a shower and get ready for work. The music "follows" him to a small speaker in the bathroom and during his shower the song he queued earlier starts playing.

In AirPlayer, movement is interpreted as a discrete measure of changes in location. As illustrated by the scenario, it allows music to follow a person around, by tracking the location of a smartphone. As the person moves in between zones, the system is able to anticipate where the user is going, thereby preparing the music in the zone(s) ahead. Similarly the system is aware of the zone a person is moving away from and stops music playback in that place. As illustrated by the scenario, not only the song playing is transferred, but also queues are transferred between zones as the user moves around.

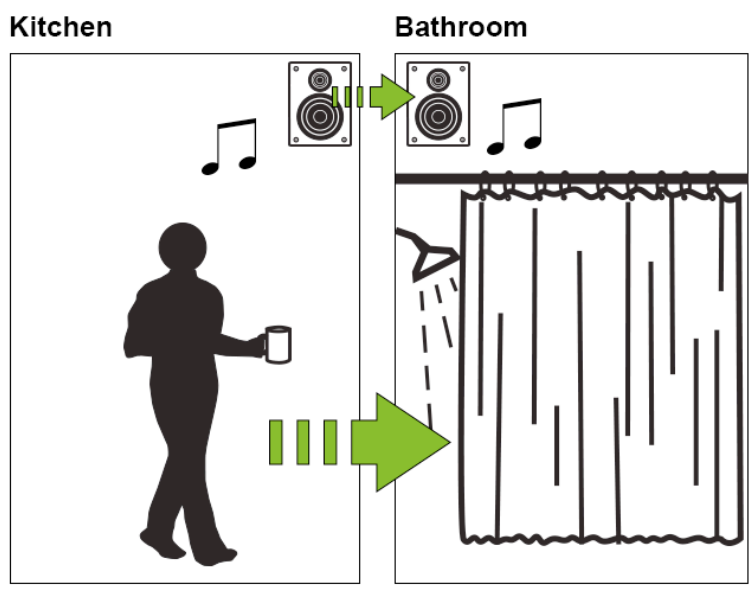

Figure 4. When the movement feature is active, music follows the user around the house automatically.

\section{Graphical User Interface}

The proxemic interaction is not a replacement for a graphical user interface (GUI) but a supplement replacing specific parts of the interaction that complicates the control of a multi-room music system. A GUI enabling the user to control music playback on a level equal to that of similar music systems is therefore necessary for the sake of conducting field evaluations.

The smartphone application provides the only GUI presented to the user. The GUI therefore both serves as a remote control for the system and a display providing visual feedback to the user. The user can control the music playback in each of the available zones through the touch input interface of the smartphone. Functionality includes queuing music on a zone, playing and pausing the music, adjusting volume etc. The functionality is kept simple and even when the proxemic features are turned off, only frequently used functionality is available. The GUI consists of three underlying screens: A music player, a queue and a music browser.

\section{Music Player}

The music player is the main screen of the application and is also the first screen the user is presented to when starting the application (Figure 5). The location and movement features can be activated/deactivated by the click of a button placed in the top bar. Once activated, the button will highlight, showing that the feature is active. When movement is active, music follows the user around between zones and naturally so does the control, i.e. the location feature is implicitly active whenever the movement feature is active. The top bar furthermore contains a link button. While the link feature is not directly related to the proxemic interaction, it is used to control the zones which the proxemic features utilise. 


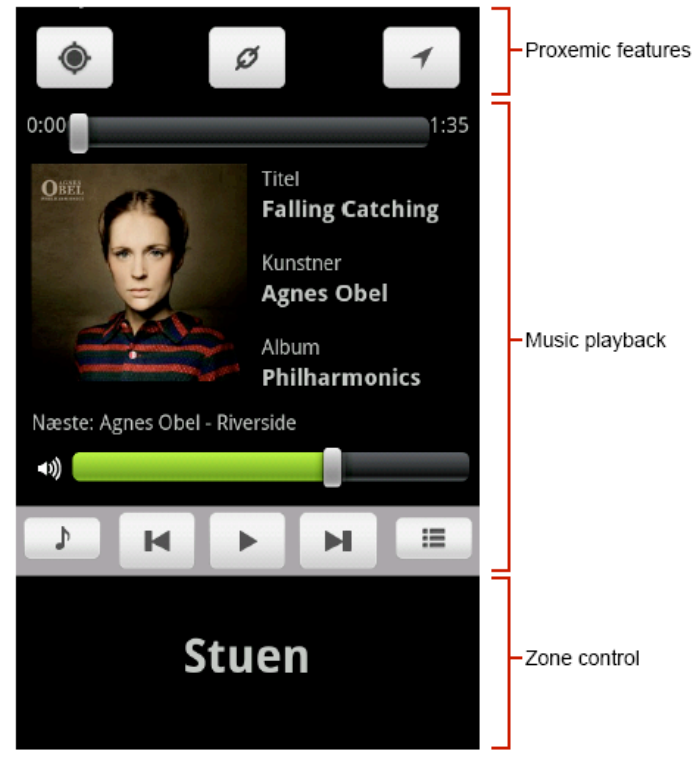

Figure 5. The music player is the point of entry and primary interaction screen of the application.

The music playback component is the main part of the music player interface and contains information and controls commonly seen in music players. The interface features a seek bar, controls to start or stop the music, skip to next or previous track, adjust volume, as well as buttons to open the browse and query screens. The music playback component additionally contains a large display of information regarding the music currently playing. The bottom of the music player is reserved for the zone indicator, which displays the name of the current zone being controlled. A swipe gesture to the right or left will manually cycle through the configured zones. When the location feature is active, it will automatically change the zone according to the location of the user.

\section{Queue}

The queue screen, shown in Figure 6, presents the current queue of songs for the active zone, as well as controls to manage the queue. The primary part of the screen is the queue of songs presented in a scrollable list. The clear button in the top right corner removes all queued songs, in the active zone, and stops playback. Controls in the bottom can be used to play a song instantly, rearrange the queue, or remove songs.

\section{Music Browser}

The music browser interface features a top bar with a back button, an add button and an add-all button. If the user presses the add button, all selected songs are added to the queue of the active zone. If the add all button is pressed, all songs on the displayed list are added to the queue. The primary part of the music browser, is a list of items from the music library, i.e. playlists, albums, artists and songs. The items are sorted alphabetically and the user can scroll the list using swipe gestures.

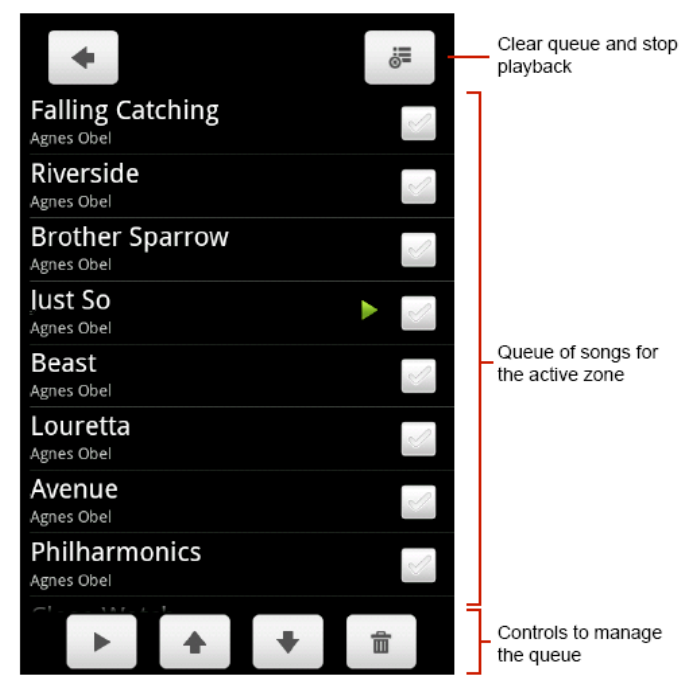

Figure 6. The queue for the active zone can be accessed and managed through the user interface.

The bottom bar of the interface contains a tabbed interface for the user to browse through the music library. For example, when the user selects the Albums tab, a list of available albums from the iTunes library is presented. From here, the user can select an album, and all songs on the album will be displayed on the list. From the song level, as shown in Figure 7, the user can select a number of songs to add to the zone queue, or simply add all songs from the album to the queue. In this way, the user can browse through the iTunes library from his smartphone, and add the desired songs to the queue of currently playing songs.

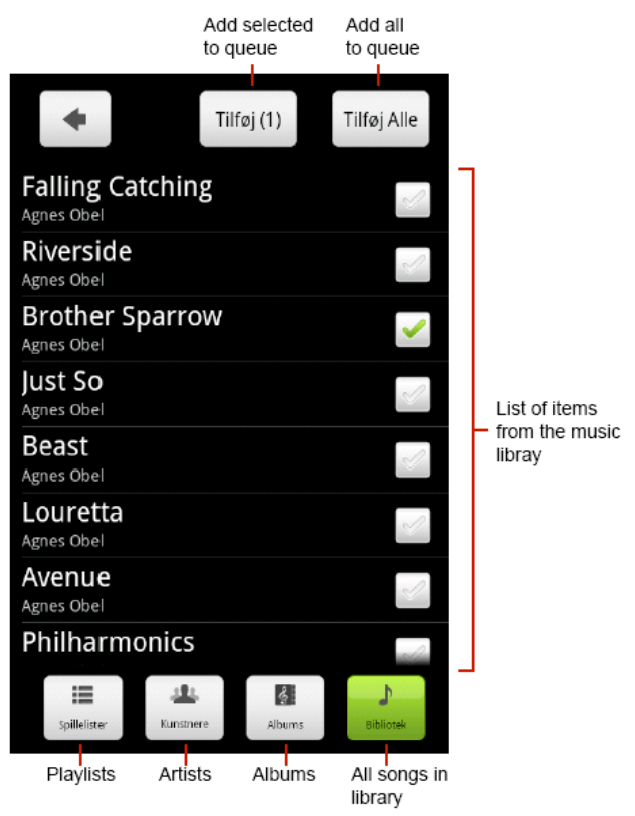

Figure 7. On the song level of the music browser, songs can be added to the queue. 


\section{SYSTEM IMPLEMENTATION}

In this section, further details about the implementation of AirPlayer are provided. This specifically includes the overall technical architecture and details about the location estimation and server application.

\section{Architecture}

The platform it builds on top of is Apple's AirPlay which allows streaming of media content between Apple products, and Apple compatible products, using a regular wireless network. The AirPlayer infrastructure relies on three types of devices: 1) A smartphone which hosts the AirPlayer client application. 2) A Mac Mini server which hosts the AirPlayer server application, the music collection, and iTunes. 3) A number of AirPort Express stations, connected to either active speakers or a stereo.

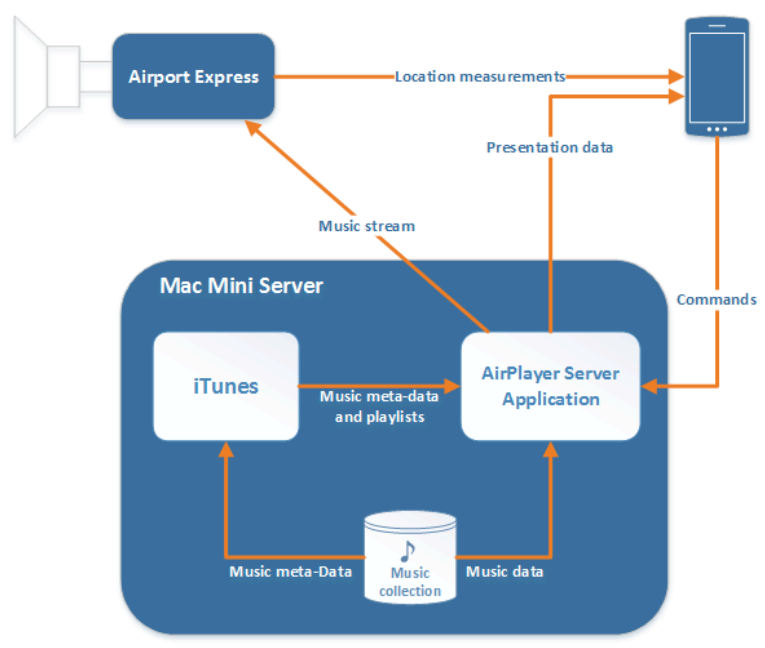

Figure 8. The overall architecture of AirPlayer consists of three types of inter-connected devices.

The AirPlayer architecture can be seen in Figure 8, which depicts relations between the components. The server application is installed on a Mac Mini server and is basically a remote controlled music player capable of streaming to AirPort Express stations. It gets the music data directly from the music collection residing on the server. Apple's music player iTunes is also installed on the server, but only serves the purpose of getting the meta-data from the music library and providing an interface where the user can manage the music collection and playlists. In addition to receive the music stream, the AirPort Express also provides location measurements handled by the smartphone application. The smartphone application communicates directly with the server application to issue commands like "skip to next song" or "move music stream to another AirPort Express". It furthermore receives data from the server, such as metadata about the currently playing song, which can be presented to the user. The user can control the system using a smartphone from anywhere in the house directly through touch input or indirectly by activating the proxemic features.

\section{Location Estimation}

The exploitation of location awareness is a central part of AirPlayer. Both the location and movement features are reliant on knowledge about the user's current position within the environment. In AirPlayer this is accomplished through a simple comparison of received signal strength indicator (RSSI) measurements, handled by the smartphone application. For the prototype application, only coarse-grained information regarding the user's position is required, i.e. in which zone the user is currently located and whether his location has changed over time. By using results from Wi-fi RSSI measurements from the AirPort Express devices, the relative location of the user can be estimated, thus creating the basis for the implementation of the proxemic interaction.

AirPlayer is a self-positioning system (Drane et al., 1997), meaning that the positioning receiver is responsible for doing appropriate measurements and uses these to position itself. In AirPlayer the mobile application is responsible for collecting the RSSI measurements, from each available AirPort Express, and use them to determine which zone it is currently in. Having the smartphone application as a self-positioning receiver makes it less dependent on changes in the infrastructure and therefore does not need to be aware of details about the setup such as the number and location of available AirPort Express stations.

\section{Server Application}

The server application is the backend of the system and is basically a remote controlled music player capable of streaming wirelessly to speakers connected to an AirPort Express. It contains information about the user's iTunes music collection including playlists. Actual management of the music, like editing playlists or reorganizing the music, is handled directly in iTunes. This provides a familiar interface for the user and results in a simpler implementation on the server side of the application.

The server application is furthermore responsible for registering and managing the configured zones. It makes use of Apple's implementation of zero configuration networking, called Bonjour, which provides a DNS based Service Discovery API that enables automatic discovery of network services. This means that every time an AirPort Express is connected to the wireless network, it will be discovered automatically by AirPlayer. The default behaviour is for each newly connected AirPort Express to create its own zone, but as mentioned, zones can be linked to form larger zones. The way it is managed in the server application is simply by letting one zone handle a number of AirPort Express connections, thereby synchronizing the control and stream of music.

\section{FIELD EVALUATION}

The field evaluation was conducted with the goal of exploring the use and challenges of an integration of proxemic interaction in a multi-room music system. 


\section{Method}

The evaluation spanned three weeks, where AirPlayer was installed and integrated with the participants' existing multi-room music system. The participants were introduced to the system in the beginning of the evaluation and would then use it in their everyday routines. Participants were asked to note their thoughts about the system in a diary. After the three weeks a semistructured interview was conducted, following the guidelines of Lazar et al. (2010). Entries from the diaries were included as a basis for a conversation regarding the system. Interviews were conducted in the homes of the participants, to provide a comfortable environment and to let the participants talk about their experiences in the place where interaction took place. Two interviewers were present at each session. One would specifically be responsible for taking notes including information about esoteric remarks, visual references etc. Interviews were furthermore recorded.

\section{Participants}

Two households, which will be referred to as A, and B, participated in the field evaluation. Both had a multiroom music system installed prior to the evaluation and were therefore familiar with the basic concept and use of such systems. The participants received a small token of appreciation for participating in the evaluation.

\section{Household A}

The first participating household had two residents. A woman aged 47 (A1) living with her 16 year old son (A2) on a small farm. The music player currently installed in their home was a Sonos system. The woman had four years of experience with the system and her son one year. Three AirPort Express stations were installed in the locations where the participants usually listened to music using their current system as illustrated in Figure 9.

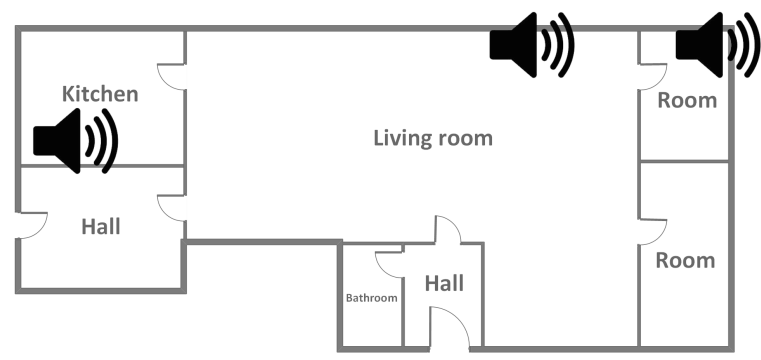

Figure 9. Floor plan of household A illustrating placement of AirPort Express stations. The floor plan only includes the part of the farm where AirPlayer was installed.

\section{Household $B$}

The second participant was a 28 year old man living alone in an apartment (B1). He already had a setup consisting of Apple products and used iTunes to play music. AirPlayer could therefore easily connect to the existing system. An additional AirPort Express was added to get more than two locations. The floor plan is shown in Figure 10.

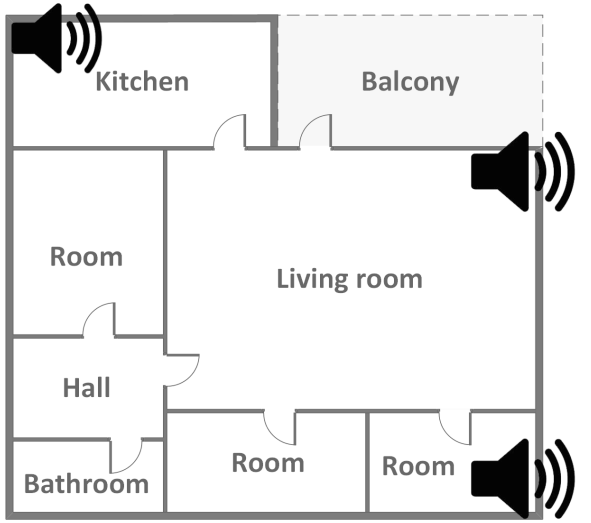

Figure 10. Floor plan of household B illustrating placement of AirPort Express stations.

\section{FINDINGS}

Basic usage was logged by the system itself, which will be presented to give an overview of the foundation for the qualitative findings.

Over the three week period household A used the system for 70 hours in total, averaging 3.3 hours per day. The system would however be used more in the first half of the evaluation period. During the 70 hours of use, the location and movement features were active for $57 \%$ and $23 \%$ respectively. The usage in household $\mathrm{B}$ had a more even distribution with a total of 43 hours, averaging 2.0 hours per day. The location feature was active $47 \%$ of the time and the movement feature 39\%. Reasons for the relatively low percentages of time the features were active, can partly be found in the findings presented later. There are however also a few low-practical explanations. One is the location feature being implicitly active when the movement feature is active, but not counted in the statistics if it is not explicitly activated in the system. Another is the fact that participants would turn features on one at a time to explore this particular feature.

From this usage, participants were able to continuously report experiences in their diary and conclusively report on their use of the system during the interview. The following findings are the result which is divided into a separate section for each proxemic interaction feature.

\section{Location}

The location feature allows the user interface of the smartphone application to automatically adapt to the zone the user is currently in. Interaction is thereby directed towards the current zone and visual feedback reflects what is playing in that particular zone. From the interviews two main findings were identified regarding simple interaction and local interaction.

\section{Simple Interaction}

The purpose of the proxemic features is to hide the complexity of interaction with the music system. Both households described the location feature as simple. They thought it was intuitive, but also very transparent. In fact, A1 was not aware that she was using it: 
To me it had to be fantastic, because I used it and I didn't even think about whether or not the feature was enabled. I actually thought it was connected with the other [feature] where music followed me from room to room. It worked when I adjusted the volume and other things. But I had not thought about the fact that I used it, but I really did. (A1)

The fact that she was not aware of having enabled the feature despite of not having it in her own Sonos system indicates that the feature was very subtle. This is especially taken into consideration that the location feature was active $57 \%$ of the time in household $\mathrm{A}$ and implicitly active when the movement feature was enabled as well. In retrospect she had no problem understanding that the feature had worked the way it did. She had simply thought of it as intuitive to use the smartphone to control the music in the room she was currently in, just as she was doing when the movement feature was active.

In household B, the participant similarly found the feature to be simple and useful. Compared to household A, he was fully aware of this feature:

As I said earlier, I think that a relatively simple feature as this one is extremely good. The fact that I do not have to find the room that I am about to play music in, makes it easy to utilise the mobile phone to control the music according to your current location. (B1)

Another point where the need for simple interaction was visible, was not in the added proxemic features but instead in missing features. Participants from both households commented that they were missing the radio or internet radio functionality of the systems they were used to. B1 also mentioned that he would have liked a larger queue size. Both of these comments indicate a need for simple interaction where it is not up to the user to constantly control the music in several locations.

\section{Local Interaction}

What we experienced from the field evaluation was that the location feature, which limits interaction to the immediate surroundings of the user, was appreciated by the participants. By enabling the location feature, they found it easy to use the remote application to control AirPlayer, as the application was aware of its current location and could therefore easily manage the music in the given zone. Both households used the location feature during the majority of the test period. As the participant from household B expresses:

I would estimate that I just used it. I just used it most of the time. Actually, I have had no real need to control another room other than the one I was present in. (B1)

This is also supported by the previous comments from A1 who utilised the location functionality without being aware of it, as she experienced that the system responded to her interactions in the zone she was currently in.

\section{Movement}

The second proxemic dimension, movement, was also implemented as a part of the interaction design of AirPlayer. When active, the movement feature allows music to follow the user around the house additionally moving the queue and control of music as well. The findings identified from the use of the movement feature are categorised as understanding background interaction and music in discrete locations.

\section{Understanding Background Interactions}

No participants initially expressed any uncertainties regarding the use of the functionality or the concept behind. When asked to describe the feature using their own words, they were able to give a concise description of how it worked. However, B1 also elaborated:

Of course, I had to get used to how it worked the first time I used it, but I do not think that we had any doubts of how it was supposed to work. (B1)

Both households had a good overall understanding of how the feature worked. Despite that, we did find indications that detailed specifics about the behaviour were not always as clear:

I would have thought that the music stopped when I went upstairs to bed. It didn't. It stayed in the last room I left - That puzzled me. (A1)

Because the music followed A1 into rooms where a speaker was present, she also expected the music to stop playing when she left these rooms. At least when she left the floor where the music system was installed. B1 did not state any problems with that, but did however in his own words also explain the feature as if it automatically turned off the music.

\section{Music in Discrete Zones}

Findings were discovered regarding the relationship between music and the subdivision into discrete zones. Both families found that the movement feature worked well, and differed from their regular music setups in the way they interact with their music system. As one of the participants from household B stated:

We have used it a lot, partly because we wanted to test the system, but also because we found it to be clever and fun to use. The thing about having the music following you is nice when you walk around at home in your own thoughts. (B1)

Household A shared this opinion towards using the feature; however they experienced slightly unintended behaviour. When asking how the feature behaved, they explained that the music should overlap for longer periods than it currently did:

It could have waited 15 seconds before it stopped the room you were leaving, and then start the music immediately as you walk into the next room. I think more overlap would have worked wonders (A1)

As participant A1 stated, she would have liked AirPlayer to have a timer based threshold instead of having one purely based on distance between two zones and the 
smartphone. The result of the behaviour was that she would turn off the feature while cleaning the house as she would move frequently between different rooms.

At times, the participants also experienced that the music would not be entirely synchronized, which were obvious when standing in the middle of two different zones with the movement feature active. The result was a slightly asynchronous playback of the same song:

As I said earlier, there were a few incidents where the music was not entirely synchronised, but we learned that we could fix it by changing the track being played and then switching back (B1)

The asynchronous behaviour was caused by technical limitations discovered in the preliminary study when the framework was developed. As B1 states, he found out that this could be solved by forcing the system to reload the songs, and thereby getting the music back in sync.

\section{DISCUSSION}

Several systems developed and evaluated in research within proxemic interaction are based on a central focal point, like a public display, where interaction takes place in the immediate surroundings. The AirPlayer prototype has been developed to explore proxemic interactions in environments that span separate locations. Focus has furthermore been on evaluating specific dimensions, namely location and movement, of proxemic interaction for a music system. In this section findings from the field evaluation are discussed.

\section{Simple Interaction}

The point of simplicity in this case is not only that the interaction is simple to understand and use, but also that the proxemic features add simplicity to the interaction with the music system. The fact that one of the participants (A1) was unaware that she had used the location feature, but still found it useful in retrospect, points out a particular property of proxemic interactions. Interaction can be very transparent to the user as long as it feels natural, and letting features become invisible to the user can even be a success criterion of the design. Results on the location feature did not indicate that it was important for the user to be informed every time the control was changed from one zone to another, or that they needed advanced features to feel more in control of the system. One reason can be that the metaphor of a single music system in each room becomes stronger with the location feature active and it feels natural to use a remote control in the room you are currently in.

\section{Local Interaction}

The main differences between multi-room music systems and traditional music players is that the music source and the control can be centralised when playing music in different locations of the home. What the location feature of AirPlayer does is really to limit the control to the room the user is currently in. This is however determined automatically. During the field evaluation of AirPlayer, the feature was well received. It was very intuitive to control music at the location of the user. Integration of wireless networks in our homes does provide great opportunities for remote controlling everything from everywhere. Our findings contribute to an ongoing argument that discrete zones within proxemic interaction can be powerful for the user to understand where interaction is directed (Marquardt et al., 2012). Not only as discrete zones surrounding a single device, but also as connected zones in a larger environment.

\section{Understanding Background Interactions}

Buxton talks about foreground and background interactions within HCI (Buxton, 1995). Where the graphical user interface of the smartphone application facilitates foreground interaction, the proxemic interactions facilitate background interactions. Making the user understand designed background interactions can be challenging. What the implementation of the movement feature showed was that although the overall concept was easy to understand, details about the interaction were difficult to make apparent to the user. One participant knew that the movement feature allowed music to follow her around and therefore also expected it to stop when she went to a room where music could not be played. As a consequence of making an effort to simplify the interaction, in our case through proxemic interactions, challenges in making details about the interaction apparent to the user are easily introduced. This is especially the case for an activity such as listening to music which is already in the background.

\section{Music in Discrete Zones}

Certain issues and unexpected behaviour were experienced by the participants in relation to the movement feature. As a consequence of the way music would move, A1 preferred not to use the movement feature while cleaning the house, as she would move frequently between rooms. The specific behaviour she experienced might be due to the layout of the house, but it does raise an interesting issue. A reason for the annoyance could be because the speakers, often placed in opposite sides of adjacent rooms, would create the feeling of the music jumping from one side of the house to the other, instead of creating a seamless experience. The location system might be divided into discrete zones, but the music is not restricted to be audible within a specific zone. This is also part of the reason why the slight asynchronous playback would be very apparent to B1, when standing in between two zones. Music additionally does not have the same requirements for the user's attention as visual content. The user can easily perceive music from two rooms at the same time. It would be interesting to explore continuous measures for movement in a ubiquitous music context.

\section{Limitations of Location System}

We are aware of the limitation imposed by the direct use of RSSI values as indication of physical distance, due to uncontrollable external influences. A more accurate indoor positioning system is however out of the scope of this paper and the focus has been on the concept of proxemic interactions in real-life contexts. What is interesting to note is that even though participants were 
asked about the usefulness of the proxemic interaction features, no comments were directly addressing problems with accuracy of the location estimation, despite the simple implementation. Other issues have surfaced during the interviews, but an overall perception of proxemic awareness and satisfaction with the features, have been experienced by the participants.

\section{CONCLUSION}

By developing a functional prototype of a multi-room music system it has been possible to evaluate implementations of the proxemic dimensions, location and movement for a multi-room music system. Specific findings on simple interaction, local interaction, understanding background interaction and music in discrete zones were identified. The findings suggest that proxemic interactions have a great potential for hiding parts of the interaction with a complex system, making it simpler to the user. It is however challenging to simplify background interactions in a way where details about the interaction are apparent. There are furthermore specific challenges in relation to music consumption as a domain. One challenge is how to handle the fact that music transcends the invisible borders of discrete zones.

\section{ACKNOWLEDGMENTS}

A special thanks goes to the field evaluation participants, and to Helle Hyllested Larsen for her work on illustrations.

\section{REFERENCES}

Ackad, C.J., Clayphan, A., Maldonado, R.M., Kay, J. Seamless and continuous user identification for interactive tabletops using personal device handshaking and body tracking. In Proc. CHI EA 2012, ACM Press (2012), 1775-1780.

Annett, M., Grossman, T., Wigdor, D., Fitzmaurice, G. Medusa: A proximity-aware multi-touch tabletop. In Proc. UIST 2011, ACM Press (2011), 337-346.

Ballendat, T., Marquardt, N., Greenberg, S. Proxemic interaction: Designing for a proximity and orientationaware environment. In Proc. ITS 2010, ACM Press (2010), 121-130.

Brumitt, B., Meyers, B., Krumm, J. EasyLiving: Technologies for intelligent environments. Handheld and Ubiquitous Computing 1927, Springer (2000), 1229.

Buxton, B., Integrating the periphery and context: A new model of telematics. In Proc. GI 1995, Canadian Information Processing Soc. (1995), 239-246

Chang, J., Kim, Y,. Context-aware application system for music playing services. In Proc. KES 2006, Springer, 76-83.

Clark, A., Dünser, A., Billinghurst, M., Piumsomboon, T., Altimira, D. Seamless interaction in space. In Proc. OZCHI 2011, ACM Press (2011), 88-97.

Drane, C., Macnaughtan, M., and Scott, C. Positioning GSM telephones. Communications Magazine, IEEE (1998) 36, 4, 46-54,59.
Greenberg, S., Marquardt, N., Ballendat, T., DiazMarino, R., Wang, M. Proxemic interactions: The new ubicomp? interactions 18, 1, ACM Press (2011), 42-50.

Hall, E.T. The Hidden Dimension. Doubleday, 1966.

Holmquist, L.E. Ubiquitous music. Interactions 12, 4 (2005), 71-ff

Ju, W., Lee, B.A., Klemmer, S.R. Range: Exploring implicit interaction through electronic whiteboard design. In Proc. CSCW 2008, ACM Press (2008), 1726.

Laibowitz, M., Gips, J., Aylward, R., Pentland, A., Paradiso, J.A. A sensor network for social dynamics. In Proc. ISPN 2006, ACM Press (2006), 483-491.

Lazar, J., Heidi, J., Hochheiser, H. Research methods in human-computer interaction. Wiley, 2010

Liikkanen, L., Amos, C., Cunningham, S.J., Downie, J.S., McDonald, D. Music interaction research in HCI: let's get the band back together. In Proc. CHI EA 2012, ACM Press (2012), 1119-1122.

Marquardt, N., Diaz-Marino, R., Boring, S., Greenberg, S. The proximity toolkit: Prototyping proxemic interactions in ubiquitous computing ecologies. In Proc. UIST 2011, ACM Press (2011), 315-326.

Marquardt, N., Greenberg, S., Informing the design of proxemic interactions. Pervasive Computing 11, 2, IEEE (2012), 14-23.

Marquardt, N., Hinckley, K., Greenberg, S., Cross-device interaction via micro-mobility and f-formations. In Proc. UIST 2012, ACM Press (2012), 13-22.

Nirjon, S., Dickerson, R.F., Li, Q., Asare, P., Stankovic, J.A., Hong, D., Zhang, B., Jiang, X., Shen, G., Zhao, F., MusicalHeart: A hearty way of listening to music. In Proc. SenSys 2012, ACM press (2012), 43-56.

Rose, M., Music in the home: Interfaces for music appliances. Personal Technologies 4, 1, Springer (2000), 45-53.

Torres-Solis, J., Falk, T.H., Chau, T. (2010). A Review of Indoor Localization Technologies: towards Navigational Assistance for Topographical Disorientation, Ambient Intelligence, Jesus, F., Molina, V. (Ed.), 2010.

Trotto, A. and Tittarelli, M. Musical viruses for graceful seduction. In Proc. NordiCHI 2012, ACM Press (2012), $731-735$

Vogel, D., Balakrishnan, R., Interactive public ambient displays: Transitioning from implicit to explicit, public to personal, interaction with multiple users. In Proc. UIST 2004, ACM Press (2004), 137-146.

Wang, M., Boring, S., Greenberg, S. Proxemic peddler: A public advertising display that captures and preserves the attention of a passerby. In Proc. PerDis 2012, ACM Press (2012) Article no. 3

Want, R., Hopper, A., Falcão, V., Gibbons, J. The active badge location system. ACM Transactions on Information Systems 10, 1, ACM Press (1992), 91-102 